\title{
Cauchy sequences and a Meir-Keeler type fixed point theorem in partial metric spaces.
}

\author{
Siditë Duraj \\ Department of Mathematics; Faculty of Natural Sciences, University of Shkodra, Shkoder, Albania \\ siditaduraj@yahoo.com \\ Elida Hoxha \\ Department of Mathematics; Faculty of Natural Sciences, University of Tirana; Tirana, Albania \\ hoxhaelida@yahoo.com
}

\begin{abstract}
In this paper we prove some new conditions for Cauchy sequences by using the diameter of orbit in partial metric spaces. A fixed point theorem for Meir-Keeler type contractions in this space is established.
\end{abstract}

Keywords: Partial metric space; Cauchy sequences; fixed point theorem; Meir-Keeler type contraction.

\section{Academic Discipline and Sub-Disciplines}

Mathematics, Functional Analysis.

\section{SUBJECT CLASSIFICATION}

Functional Analysis

\section{Introduction.}

The notion of a partial metric space was introduced by G.S. Metthews [10,11] in 1992. The partial metric space is a generalization of the usual metric spaces in which the distance of a point from itself may not be zero. Recently, many authors have been focused on the partial metric spaces and its topological properties. $[1,12,13]$.They show that partial metric spaces have many applications both in mathematics and computer science [8, 13]. The concept of Cauchy sequences is very important in functional analysis and especially in fixed point theory.

In [4] we obtained some conditions for equivalent Cauchy sequences and 0 -equivalent 0 -Cauchy sequences in partial metric spaces.

The Banach contraction principle [14] is the most celebrated fixed point theorem. It is very useful, simple, and classical tool in nonlinear analysis. This principle has many generalizations. For example, in 1969 [2] Meir and Keeler proved a fixed point theorem for the mappings satisfying a $(\varepsilon-\delta)$ contractive condition. Some generalizations of Meir-Keeler fixed point theorem (see 9, 5,6) established a class of the contractions called the Mier-Keeler type contraction.

In this paper we will show some conditions about Cauchy sequences in partial metric spaces establish a fixed point theorem for a Meir- Keeler type contraction in these spaces.

\section{Preliminaries.}

For convenience we start with the following definitions, lemmas, and theorems.

Definition 1. [10] A function $p: X \times X \rightarrow R^{+}$is a partial metric on $X$ if, for all $x, y, z \in X$, the following conditions hold:

$\left.\mathrm{p}_{1}\right) x=y$ if and only if $p(x, x)=p(x, y)=p(y, y)$,

$\left.\mathrm{p}_{2}\right) p(x, x) \leq p(x, y)$

p $) p(x, y)=p(y, x)$,

p $) \quad p(x, y) \leq p(x, z)+p(z, y)-p(z, z)$

In this case, the pair $(X, p)$ is called a partial metric space.

It is clear that if $p(x, y)=0$ then from $\left(p_{1}\right)$ and $\left(p_{2}\right), x=y$. But, if $x=y, p(x, y)$ may not be 0 . As an example of partial metric space we have, $\left(R^{+}, p\right)$ where $p(x, y)=\max \{x, y\}$.

Each partial metric $p$ on $X$ generates a $T_{0}$-topology on $X$, which has as base the family of open $p$-balls $\left\{B_{p}(x, \varepsilon): x \in X, \varepsilon>0\right\}$, where $B_{p}(x, \varepsilon)=\{y \in X: p(x, y)<\varepsilon+p(x, x)\}$ for all $x \in X$ and $\varepsilon>0$

Definition 2. $[10,11]$ A sequence $\left\{x_{n}\right\}$ in a partial metric space $(X, p)$ is said to be: 
(i) $p$-convergent to a point $x \in X$ if $\lim _{n \rightarrow \infty} p\left(x, x_{n}\right)=p(x, x)$;

(ii) $p$-Cauchy sequence if $\lim _{n, m \rightarrow \infty} p\left(x_{m}, x_{n}\right)$ exists and is finite.

Notice that the limit of sequence in partial metric space is not necessary unique.

Proposition 3. [11] Every partial metric $p$ defines a metric $d_{p}$, where

$d_{p}(x, y)=2 p(x, y)-p(x, x)-p(y, y)$ for all $x, y \in X$.

The metric $d_{p}$ is called the metric associated with partial metric $p$.

Lemma 1. $[10,11]$

(1) A sequence $\left\{x_{n}\right\}$ is a $p$-Cauchy sequence in a partial metric space $(X, p)$ if and only if it is a Cauchy sequence in the metric space $\left(X, d_{p}\right)$.

(2) $(X, p)$ is complete if and only if the metric space $\left(X, d_{p}\right)$ is complete.

Lemma 2. [7] Let $(X, p)$ be a partial metric space and let $\left(x_{n}\right)$ and $\left(y_{n}\right)$ be sequences in $\mathrm{X}$ such that $x_{n} \rightarrow x$ and $y_{n} \rightarrow y$ with respect to $d_{p}$. Then $\lim _{n \rightarrow \infty} p\left(x_{n}, y_{n}\right)=p(x, y)$

Definition 4. The sequences $\left(x_{n}\right)$ and $\left(y_{n}\right)$ in a metric space $(X, d)$ are called equivalent if $\lim _{n \rightarrow \infty} d\left(x_{n}, y_{n}\right)=0$.

Definition 5. The sequences $\left(x_{n}\right)$ and $\left(y_{n}\right)$ in a partial metric space $(X, p)$ are called equivalent if $\lim _{n \rightarrow \infty} p\left(x_{n}, y_{n}\right)$ exists and is finite.

Definition 6. The sequences $\left(x_{n}\right)$ and $\left(y_{n}\right)$ in a partial metric space $(X, p)$ are called equivalent Cauchy if they are Cauchy and equivalent in $(X, p)$.

Definition 7. Let $(X, p)$ be a partial metric space. A sequence $\left\{x_{n}\right\}$ in $\mathrm{X}$ is called 0 -Cauchy if $\lim _{n, m \rightarrow \infty} p\left(x_{m}, x_{n}\right)=0$

Definition 8. The sequences $\left(x_{n}\right)$ and $\left(y_{n}\right)$ in a partial metric space $(X, p)$ are called 0-equivalent if $\lim _{n \rightarrow \infty} p\left(x_{n}, y_{n}\right)=0$.

Definition 9. The sequences $\left(x_{n}\right)$ and $\left(y_{n}\right)$ in a partial metric space $(X, p)$ are called 0-equivalent 0-Cauchy if they are 0 -Cauchy and 0 -equivalent in $(X, p)$.

Definition 10. Let $(X, p)$ be a partial metric space

i) A subset $\mathrm{A}$ in $\mathrm{X}$ is called bounded if there exists a real number $\mathrm{M}>0$ such that $p(x, y) \leq M$ for all $x, y \in A$;

ii) If $A$ is bounded set of $X$, then the diameter of $A$ is denoted by $\delta(A)$ and is defined by $\delta(A)=\sup \{p(x, y) ; x, y \in A\}$

Theorem 2.2.[4] If the sequences $\left(x_{n}\right)$ and $\left(y_{n}\right)$ are equivalent Cauchy in $\left(X, d_{p}\right)$, then they are equivalent Cauchy in partial metric space $(X, p)$.

The example 3 in [4] shows that the converse of the theorem 2.2 is not true.

Also, in [4] we proved some new conditions for equivalent Cauchy sequences in partial metric spaces as follows:

Theorem 2.1. [4] Let $(X, p)$ be a partial metric space and $\left(x_{n}\right),\left(y_{n}\right)$ two sequences in it. If the sequences $\left(x_{n}\right)$ , $\left(y_{n}\right)$ satisfy one of the following conditions, then the sequences $\left(x_{n}\right),\left(y_{n}\right)$ are equivalent Cauchy in $(X, p)$. 
(1) The sequences $\left(x_{n}\right)$ and $\left(y_{n}\right)$ are bounded in $(X, p)$ and

$\forall \varepsilon>0, \exists r \in N, \exists \delta \in(0,+\infty), \exists \varepsilon_{0} \in(0, \varepsilon)$ such that $\delta_{i j} \leq \varepsilon+\delta \Rightarrow p\left(x_{i+r}, y_{j+r}\right) \leq \varepsilon_{0}$ whenever $i, j \in N$

(2) The sequences $\left(x_{n}\right)$ and $\left(y_{n}\right)$ are bounded in $(X, p)$ and

$\forall \varepsilon>0, \exists r \in N, \exists \delta \in(0,+\infty)$ such that $\delta_{i j} \leq \varepsilon+\delta \Rightarrow \delta_{i+r, j+r}<\varepsilon$, whenever $i, j \in N$

(3) The sequences $\left(x_{n}\right)$ and $\left(y_{n}\right)$ are bounded in $(X, p)$ and

$\forall n \in N, \exists \alpha_{n} \in(0,+\infty), \exists r \in N$, such that $\delta_{i j}<\alpha_{n} \Rightarrow \delta_{i+r, j+r}<\frac{1}{n}$ whenever $i, j \in N$

(4) The sequences $\left(x_{n}\right)$ and $\left(y_{n}\right)$ are bounded in $(X, p)$ and

$\forall \varepsilon>0, \exists r \in N, \exists \delta \in(0,+\infty), \exists \varepsilon_{0} \in(0, \varepsilon)$ such that $\delta_{i j} \leq \varepsilon+\delta \Rightarrow \delta_{i+r, j+r} \leq \varepsilon_{0}$ whenever $i, j \in N$

These conditions in theorem 2.1 are necessary and sufficient for 0 -equivalent 0 -Cauchy sequences in partial metric spaces as the following theorem shows.

Definition 8. [ 7] Let $(X, d)$ be a partial metric space and T a self-mapping of $X$.

1. $T$ is called orbitally continuous if

$\lim _{i, j \rightarrow \infty} p\left(T^{n_{i}} x, T^{n_{j}} x\right)=\lim _{i, j \rightarrow \infty} p\left(T^{n_{i}} x, z\right)=p(z, z)$ implies $\lim _{i, j \rightarrow \infty} p\left(T T^{n_{i}} x, T T^{n_{j}} x\right)=\lim _{i, j \rightarrow \infty} p\left(T T^{n_{i}} x, T z\right)=p(T z, T z)$

for each $x \in X$.

Equivalently, $\mathrm{T}$ is orbitally continuous provided that if $T^{n_{i}} x \rightarrow z$ in $\left(X, d_{p}\right)$, then $T^{n_{i}+1} x \rightarrow T z$ in $\left(X, d_{p}\right)$ for each $x \in X$.

Theorem 2.2. [2] ( Fixed point theorem of Meir-Keler) Let $(X, d)$ be a metric space and let T be a mapping from $X$ into itself satisfying the following condition:

$\forall \varepsilon>0, \exists \delta(\varepsilon)>0$ such that $\varepsilon \leq d(x, y)<\varepsilon+\delta(\varepsilon) \Rightarrow d(T x, T y)<\varepsilon$

Then T has a unique fixed point $z \in X$. Moreover, for all $x \in X$, the sequence $\left\{T^{n} x\right\}$ converges to $z$.

\section{Main Results.}

Let $\left(x_{n}\right)$ be a sequence in partial metric space $(X, p)$.

Define $\delta_{i j}\left(\left(x_{n}\right)\right)=\sup \left\{p\left(x_{m}, x_{k}\right): m \geq i, k \geq j\right\} \forall(i, j) \in N^{2}$.

Preposition 3.1. Let $(X, p)$ be a partial metric space and $\left(x_{n}\right)$ a sequence in it. If one $\delta_{i_{0} j_{0}}\left(\left\{x_{n}\right\}\right)$ is finite than all $\delta_{i j}\left(\left\{x_{n}\right\}\right)$ are finite.

Proof. Denote $A=\max \left\{p\left(x_{m}, x_{i_{0}}\right), 1 \leq m \leq i_{0}\right\}$ and $B=\max \left\{p\left(x_{k}, x_{j_{0}}\right) 1 \leq k \leq j_{0}\right\}$

The proof is similar with the proof of preposition 5 in [4] replacing $y_{k}$ with $x_{k}$.

Corollary 3.2. Let $(X, p)$ be a partial metric space and $\left(x_{n}\right)$ a sequence in it. The sequences $\left(x_{n}\right)$ is bounded if and only if $\delta_{11}\left(\left\{x_{n}\right\}\right)$ is finite.

The proof is similar with the proof of Corollary 6 in [4] replacing $y_{k}$ with $x_{k}$.

Theorem 3.3. Let $(X, p)$ be a partial metric space and $\left(x_{n}\right)$ a sequence in it. If the sequences $\left(x_{n}\right)$ satisfies one of the following conditions, then the sequence $\left(x_{n}\right)$ is Cauchy in $(X, p)$. 
(1) The sequences $\left(x_{n}\right)$ is bounded in $(X, p)$ and $\forall \varepsilon>0, \exists r \in N, \exists \delta \in(0,+\infty), \exists \varepsilon_{0} \in(0, \varepsilon)$ such that $\delta_{i j}\left(\left\{x_{n}\right\}\right) \leq \varepsilon+\delta \Rightarrow p\left(x_{i+r}, x_{j+r}\right) \leq \varepsilon_{0}$ whenever $i, j \in N$

(2) The sequences $\left(x_{n}\right)$ is bounded in $(X, p)$ and

$\forall \varepsilon>0, \exists r \in N, \exists \delta \in(0,+\infty)$ such that $\delta_{i j}\left(\left\{x_{n}\right\}\right) \leq \varepsilon+\delta \Rightarrow \delta_{i+r, j+r}\left(\left\{x_{n}\right\}\right)<\varepsilon$, whenever $i, j \in N$

(3) The sequences $\left(x_{n}\right)$ is bounded in $(X, p)$ and

$\forall n \in N, \exists \alpha_{n} \in(0,+\infty), \exists r \in N$, such that $\delta_{i j}\left(\left\{x_{n}\right\}\right)<\alpha_{n} \Rightarrow \delta_{i+r, j+r}<\frac{1}{n}$ whenever $i, j \in N$

(4) The sequences $\left(x_{n}\right)$ is bounded in $(X, p)$ and

$\forall \varepsilon>0, \exists r \in N, \exists \delta \in(0,+\infty), \exists \varepsilon_{0} \in(0, \varepsilon)$ such that $\delta_{i j}\left(\left\{x_{n}\right\}\right) \leq \varepsilon+\delta \Rightarrow \delta_{i+r, j+r}\left(\left\{x_{n}\right\}\right)<\varepsilon_{0}$ whenever $i, j \in N$

\section{Proof.}

Let $\left(x_{n}\right)$ be a sequence in $(X, p)$ satisfying (1). Define

$\alpha_{n}=\delta_{n, n}=\sup \left\{p\left(x_{i}, x_{j}\right), i \geq n, j \geq n\right\}$

The sequences $\left(\alpha_{n}\right)$ is decreasing and positive. Hence it converges and $\lim _{n \rightarrow \infty} a_{n}=\inf \left\{\alpha_{n}: n \in N\right\}=a \geq 0$

Suppose that $a>0$. From the condition (1) for $\varepsilon=a>0$ there are $r \in N, \varepsilon_{0} \in(0, \varepsilon)$ and $\delta>0$

such that $\delta_{i j}\left(\left\{x_{n}\right\}\right) \leq \varepsilon+\delta \Rightarrow p\left(x_{i+r}, x_{j+r}\right) \leq \varepsilon_{0}$ whenever $i, j \in N$

For this $\delta>0$ exists $p \in N$ such that for $n \geq p \Rightarrow \alpha_{n}<a+\delta=\varepsilon+\delta$

For $i \geq p, j \geq p$ we have $\delta_{i j}\left(\left\{x_{n}\right\}\right) \leq \alpha_{p}=\delta_{p, p}<\varepsilon+\delta$.By (5) we have $p\left(x_{i+r}, x_{j+r}\right) \leq \varepsilon_{0}$.

But it is obvious that $i+r=k \geq p+r, j+r=l \geq p+r$, so $p\left(x_{k}, x_{l}\right) \leq \varepsilon_{0}<\varepsilon=a$, which is a contradiction. Hence we have $\lim _{n \rightarrow \infty} a_{n}=\inf \left\{\alpha_{n}: n \in N\right\}=0$. But $p\left(x_{i}, x_{j}\right) \leq a_{\min \{i, j\}}$

and whereas $\lim _{n \rightarrow \infty} a_{n}=0$ we have $\lim _{i, j \rightarrow \infty} p\left(x_{i}, x_{j}\right)=0$. So the sequence $\left(x_{n}\right)$ is Cauchy.

Furthermore, since $p\left(x_{n}, x_{n}\right) \leq \alpha_{n}$ and $\lim _{n \rightarrow \infty} a_{n}=0$ hold, then $\lim _{n \rightarrow \infty} p\left(x_{n}, x_{n}\right)=0$.

(2) Let $\left(x_{n}\right)$ be a sequence in $(X, p)$ satisfying (2).

As in theorem 7 in [4], we first shall prove that (2) $\Rightarrow(3)$ and if $\left(x_{n}\right)$ is satisfying (3) in the same way as in (1) above, we can prove that the sequence $\left(x_{n}\right)$ is Cauchy in $(X, p)$..

(4). Let $\left(x_{n}\right)$ be a sequence in $(X, p)$ satisfying (4).

It is clear that $(4) \Rightarrow(2)$ and by (2) immediately follows that the sequences $\left(x_{n}\right)$ is Cauchy in $(X, p)$.

Remark 3.4. The converse of the theorem 3.3 is not true. For this we can see the following example.

Example 3.5. Let $\mathrm{X}=\mathrm{R}^{+}$and define a mapping $p: R x R \rightarrow R^{+}$by $p(x, y)=\max \{x, y\}$ as a partial metric.

The sequence $\left(x_{n}\right)=\left(\frac{1}{2}-\frac{1}{n}\right)$ is Cauchy in $(X, p)$. But, $\delta_{i j}=\frac{1}{2}$ for $i, j \in N$ and for $\varepsilon=\frac{1}{2}$, for any $\delta>0$ and $r>0$, though $\delta_{i j}=\frac{1}{2}<\varepsilon+\delta$ we have $\delta_{i+r, j+r}=\frac{1}{2} \geq \varepsilon$. 
So, the sequence $\left(y_{n}\right)=\left(\frac{1}{2}-\frac{1}{n}\right)$ do not satisfy the condition (2).

In the same way we can show that this sequence do not satisfy and the conditions (1), (3) and (4).

But if $\left(x_{n}\right)$ is 0 -Cauchy sequence then the converse of the theorem 3.3 is true and we can prove the following theorem.

Theorem 3.6. Let $(X, p)$ be a partial metric space and $\left(x_{n}\right)$ a sequence in it. The sequence $\left(x_{n}\right)$ is 0 -Cauchy sequence in $(X, p)$ if and only if it satisfies one of the following conditions,

(1) The sequences $\left(x_{n}\right)$ is bounded in $(X, p)$ and

$\forall \varepsilon>0, \exists r \in N, \exists \delta \in(0,+\infty), \exists \varepsilon_{0} \in(0, \varepsilon)$ such that $\delta_{i j}\left(\left\{x_{n}\right\}\right) \leq \varepsilon+\delta \Rightarrow p\left(x_{i+r}, x_{j+r}\right) \leq \varepsilon_{0}$ whenever $i, j \in N$

(2) The sequences $\left(x_{n}\right)$ is bounded in $(X, p)$ and

$\forall \varepsilon>0, \exists r \in N, \exists \delta \in(0,+\infty)$ such that $\delta_{i j}\left(\left\{x_{n}\right\}\right) \leq \varepsilon+\delta \Rightarrow \delta_{i+r, j+r}\left(\left\{x_{n}\right\}\right)<\varepsilon$, whenever $i, j \in N$

(3) The sequences $\left(x_{n}\right)$ is bounded in $(X, p)$ and

$\forall n \in N, \exists \alpha_{n} \in(0,+\infty), \exists r \in N$, such that $\delta_{i j}\left(\left\{x_{n}\right\}\right)<\alpha_{n} \Rightarrow \delta_{i+r, j+r}<\frac{1}{n}$ whenever $i, j \in N$

(4) The sequences $\left(x_{n}\right)$ is bounded in $(X, p)$ and

$\forall \varepsilon>0, \exists r \in N, \exists \delta \in(0,+\infty), \exists \varepsilon_{0} \in(0, \varepsilon)$ such that $\delta_{i j}\left(\left\{x_{n}\right\}\right) \leq \varepsilon+\delta \Rightarrow \delta_{i+r, j+r}\left(\left\{x_{n}\right\}\right)<\varepsilon_{0}$ whenever $i, j \in N$

\section{Proof.}

By the proof of the theorem 3.3 if the sequence $\left(x_{n}\right)$ satisfies one of the conditions (1)-(4) it is Cauchy sequence and $\lim _{i, j \rightarrow \infty} p\left(x_{i}, x_{j}\right)=\lim _{i \rightarrow \infty} p\left(x_{i}, x_{i}\right)=\lim _{i \rightarrow \infty} p\left(x_{j}, x_{j}\right)=0$. So the sequence $\left(x_{n}\right)$ is 0-Cauchy sequence in $(X, p)$.

Conversely, if $\left(x_{n}\right)$ is a 0 -Cauchy sequence in $(X, p)$, then it is a Cauchy sequence with respect to $d_{p}$. So, by Definition 1 and 7 , we have

$\lim _{i, j \rightarrow \infty} d_{p}\left(x_{i}, x_{j}\right)=\lim _{i \rightarrow \infty}\left[2 p\left(x_{i}, x_{j}\right)-p\left(x_{i}, x_{i}\right)-p\left(x_{j}, x_{j}\right)\right]=0$

Therefore, $\left(x_{n}\right)$ is Cauchy in metric space $\left(X, d_{p}\right)$ and as shown in [3] the conditions (1), (2), and (4) are equivalent to being of sequence $\left(x_{n}\right)$ Cauchy sequence in metric space.

So, now we can prove that if sequence $\left(x_{n}\right)$ is 0 -Cauchy in $(X, p)$, then it satisfies the condition (3).

By the definition 1 and 7, we have $\lim _{i, j \rightarrow \infty} p\left(x_{i}, x_{j}\right)=\lim _{i \rightarrow \infty} p\left(x_{i}, x_{i}\right)=\lim _{i \rightarrow \infty} p\left(x_{j}, x_{j}\right)=0$.

Then, for $n \in N$ there is $P \in N$ such that for $i>P, j>P$ we have $p\left(x_{i}, x_{j}\right)<\frac{1}{n}$ and so $\delta_{P P}<\frac{1}{n}$.

Hence, for $\alpha_{n}>\frac{1}{n}, r=P$ we have $\delta_{i j}<\alpha_{n} \Rightarrow \delta_{i+r, j+r}<\delta_{P P}<\frac{1}{n}$ whenever $i, j \in N$. So (3) hold.

Let $(X, p)$ be a partial metric space and T a self-mapping define on $\mathrm{X}$. For each $x \in X$, we define the orbit of $\mathrm{T}$ by $O(x)=\left\{x, T x, T^{2} x, T^{3} x,, T^{n} x,,,\right\} \quad$ and $\delta_{i j}=\sup \left\{p\left(T^{m} x, T^{k} y\right): m \geq i, k \geq j\right\} \forall(i, j) \in N^{2}$.

Theorem 3.6. Let $(X, p)$ be a complete partial metric space and T a self-mapping orbitally continuous define on X. If T satisfies one of the following condition, than T has a unique fixed point $z \in X$ Moreover, $\lim _{n \rightarrow \infty} T^{n} x=z$ for any $x \in X$.

(1) For all $x, y \in X$, the sequences $\left(T^{i} x\right)$ and $\left(T^{j} y\right)$ are bounded in $(X, p)$ and 
$\forall \varepsilon>0, \exists r \in N, \exists \delta \in(0,+\infty), \exists \varepsilon_{0} \in(0, \varepsilon)$ such that $\delta_{i j} \leq \varepsilon+\delta \Rightarrow p\left(T^{i+r} x, T^{j+r} y\right) \leq \varepsilon_{0}$ whenever $i, j \in N$

(2) For all $x, y \in X$, the sequences $\left(T^{i} x\right)$ and $\left(T^{j} y\right)$ are bounded in $(X, p)$ and $\forall \varepsilon>0, \exists r \in N, \exists \delta \in(0,+\infty)$ such that $\delta_{i j} \leq \varepsilon+\delta \Rightarrow \delta_{i+r, j+r}<\varepsilon$, whenever $i, j \in N$

(3) For all $x, y \in X$, the sequences $\left(T^{i} x\right)$ and $\left(T^{j} y\right)$ are bounded in $(X, p)$ and $\forall n \in N, \exists \alpha_{n} \in(0,+\infty), \exists r \in N$, such that $\delta_{i j}<\alpha_{n} \Rightarrow \delta_{i+r, j+r}<\frac{1}{n}$ whenever $i, j \in N$

(4) For all $x, y \in X$, the sequences $\left(T^{i} x\right)$ and $\quad\left(T^{j} y\right)$ are bounded in $\quad(X, p)$ and $\forall \varepsilon>0, \exists r \in N, \exists \delta \in(0,+\infty), \exists \varepsilon_{0} \in(0, \varepsilon)$ such that $\delta_{i j} \leq \varepsilon+\delta \Rightarrow \delta_{i+r, j+r} \leq \varepsilon_{0}$ whenever $i, j \in N$

Prof. Let $x \in X$. We define the iterative sequence $\left\{x_{n}\right\}$ as follows $x_{n+1}=T x_{n}$, for $n \in N$.

If there exists $n_{o} \in N$ such that $x_{n_{0}}=x_{n_{0}+1}$ than $x_{n_{0}}$ is a fixed point of T. Assume then that $x_{n} \neq x_{n+1}$ for each $n \in N$.

We first shall prove that if T satisfy one of the conditions (1)- (4) the sequence $\left\{x_{n}\right\}$ is a Chauchy sequence.

(1) Suppose T satisfies the condition (1). Substituting $x=x_{n}$ and $y=x_{n+1}$ in (1) we obtain:

the sequence $\left\{x_{n}\right\}$ is bounded in $(X, p)$ and

$\forall \varepsilon>0, \exists r \in N, \exists \delta \in(0,+\infty), \exists \varepsilon_{0} \in(0, \varepsilon)$ such that $\delta_{i j} \leq \varepsilon+\delta \Rightarrow p\left(T^{i+r} x, T^{j+r} y\right) \leq \varepsilon_{0}$ whenever $i, j \in N$

but $\delta_{i j}=\sup \left\{p\left(T^{m} x, T^{k} y\right): m \geq i, k \geq j\right\}=\sup \left\{p\left(T^{m} x_{n}, T^{k} x_{n+1}\right): m \geq i, k \geq j\right\}=\sup \left\{p\left(T^{m+n} x, T^{k+n+1} x\right): m \geq i, k \geq j\right\}$

$=\sup \left\{p\left(x_{m+n}, x_{k+1+n}\right): m \geq i, k \geq j\right\}=\delta_{i+n, j+n+1}\left(\left\{x_{n}\right\}\right)$

and $p\left(T^{i+r} x_{n}, T^{j+r} x_{n+1}\right)=p\left(x_{n+i+r}, x_{n+1+j+r}\right)$.

So the sequence $\left\{x_{n}\right\}$ satisfies condition (1) in theorem 3 , so it is a Cauchy sequence in $(X, p)$.

(2) Suppose T satisfies the condition (2). We first shall prove that (2) $\Rightarrow(3)$.

For $n \in N$, take $\varepsilon=\frac{1}{n}$ and by (2) we have that exists $r \in N, \delta>0$ and $\alpha_{n}=\frac{1}{n}+\delta$ such that $\delta_{i j} \leq \varepsilon+\delta=\alpha_{n} \Rightarrow \delta_{i j}<\varepsilon=\frac{1}{n}$ for $i, j \in N$

(3) Now, suppose T satisfies (3). In the same way as (1), substituting $x=x_{n}$ and $y=x_{n+1}$ in (3) we obtain: the sequence $\left\{x_{n}\right\}$ is bounded in $(X, p)$ and $\forall n \in N, \exists \alpha_{n} \in(0,+\infty), \exists r \in N$, such that $\delta_{i j} \leq \alpha_{n} \Rightarrow \delta_{i+r, j+r} \leq \frac{1}{n}$ whenever $i, j \in N$ but $\delta_{i j}=\sup \left\{p\left(T^{m} x, T^{k} y\right): m \geq i, k \geq j\right\}=\sup \left\{p\left(T^{m} x_{n}, T^{k} x_{n+1}\right): m \geq i, k \geq j\right\}=\sup \left\{p\left(T^{m+n} x, T^{k+n+1} x\right): m \geq i, k \geq j\right\}$ $=\sup \left\{p\left(x_{m+n}, x_{k+1+n}\right): m \geq i, k \geq j\right\}=\delta_{i+n, j+n+1}\left(\left\{x_{n}\right\}\right)$ and $\delta_{i+r, j+r}=\sup \left\{p\left(T^{m} x, T^{k} y\right): m \geq i+r, k \geq j+r\right\}=\delta_{i+n,+r j+n+1+r}\left(\left\{x_{n}\right\}\right)$

So the sequence $\left\{x_{n}\right\}$ satisfies condition (3) in theorem 3 , so it is a Cauchy sequence in $(X, p)$.

(4) It is clear that $(4) \Rightarrow(2)$ and if T satisfies (4) than by (2) the sequence $\left\{x_{n}\right\}$ is a Cauchy sequence in $(X, p)$.

Now, since $\left\{x_{n}\right\}$ is a Cauchy sequence in $(X, p)$, by Lemma 1 , it is a Cauchy sequence in the metric space $\left(X, d_{p}\right)$. Since $(X, p)$ is complete, by Lemma 2, it is complete with respect to metric $d_{p}$, so there is $z \in X$ such that $x_{n} \rightarrow z$ 
with respect to $d_{p}$. By the orbital continuity of $\mathrm{T}$, we deduce that $x_{n} \rightarrow T z$ with respect to metric $d_{p}$. Hence $z=T z$ and $z$ is a fixed point of $T$.

Let $y \in X$, where $y \neq x$. The iterative sequence $\left\{y_{n}\right\}$, where $y_{n+1}=T y_{n}$, for $n \in N$ is a Cauchy sequence in ( $X, p$ ) and $y_{n} \rightarrow z_{1}$

The sequences $\left\{x_{n}\right\}$ and $\left\{y_{n}\right\}$ satisfy conditions (1)-(4) in theorem 2.1, so they are equivalent Cauchy sequences in $(X, p)$ and as shown in the proof of the theorem 2.1. in [4] we have

$\lim _{n \rightarrow \infty} p\left(x_{n}, x_{n}\right)=\lim _{n \rightarrow \infty} p\left(x_{n}, y_{n}\right)=\lim _{n \rightarrow \infty} p\left(y_{n}, y_{n}\right)$.

Also, whereas the sequences $\left\{x_{n}\right\}$ and $\left\{y_{n}\right\}$ converge to $z$ and $z_{1}$ respectively with respect to $d_{p}$, by Lemma 2, we have $p\left(z, z_{1}\right)=\lim _{n \rightarrow \infty} p\left(x_{n}, y_{n}\right)=0$ and consequently $z=z_{1}$, which concludes the proof.

\section{REFERENCE}

[1] Altun.I, Sola. F, Simsek. H, Generalized contractions on partial metric spaces. Topology Appl. 157(18), 2010, 27782785.

[2] A. Meir and E. Keeler, A theoremon contraction mappings, J.Math.Anal.Appl. 28(1969), 326-329.1,3

[3] Bushati. S,. Disa pohime për vargjet Koshi. Bul.Shk. Univ. Shkodrës, Nr. 53(4), 2001, 5-7.

[4] Duraj. S, Hoxha.E, The equivalent Cauchy sequences in partial metric spaces, Journal of Advances in Mathematics (JAM), vol 12, nr.4, 2016, 6148-6155.

[5] J. Jachymski, Equivalent conditions and the Meir-Keeler type theorems, J.Math.Anal.Appl. 194, (1995), 293-303.1

[6] J. Matkowski, Fixed point theorems for contractive mappings in metric spaces, Casopis Pro Pestovani Matematiky. 105 94), (1980), 341-344.1

[7] Karapinar.E, Romaguera. S, Nonunique fixed point theorems in partial metric spaces. Published by Fac. of Scie. and Math., Univ. of Nis, Serbia, 2013, 1305-1314.

[8] Kopperman. R.,Matthews. S.G, and Pajoohesh .H.: What do partial metrics represent?, Spatial representation: discrete vs. continuous computational models, Dagstuhl Seminar Proceedings, No. 04351, Internationales Begegnungs- und Forschungszentrumf “ ur Informatik (IBFI), Schloss Dagstuhl, Germany, (2005).

[9] Lj. B. Ciric, A new fixed point theorem for contractivemappings, Publ. Inst. Math. (Beograd) 30 (44), (1981), 25-27.1

[10] Matthews. S. G., Partial metric topology, Research Report 212, Dept. of Computer Science, University ofWarwick, (1992).

[11] Matthews. S. G., Partial metric topology, Proc. 8th Summer Conference on General Topology and Applications, Ann. New YorkAcad. Sci. 728 (1994). 183-197.

[12] Oltra.S. and Valero. O., Banach's fixed point theorem for partial metric spaces, Rend. Ist. Mat. Univ. Trieste 36 (2004), 17-26.

[13] Romaguera. S. and Valero.O, A quantitative computational model for complete partial metric spaces via formal balls, Math.Struct. Comp. Sci. 19 (2009), 541-563. 


\section{AUTHOR's ProfiLe}

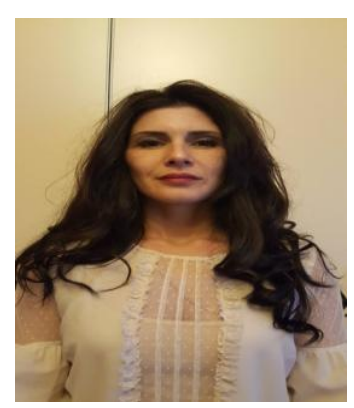

\section{M.Sc. Siditë Duraj (DOB-19/09/1980)}

Completed her M.Sc in Mathematics at Tirana University in 2011, she is a Ph. D student. She works as a lecturer at Shkodra University, in the Department of Mathematics, Faculty of Natural Science, Albania for more than 5 years. Her subject of teaching is Mathematical Analysis and Functional Analysis. Her research field is Functional Analysis.

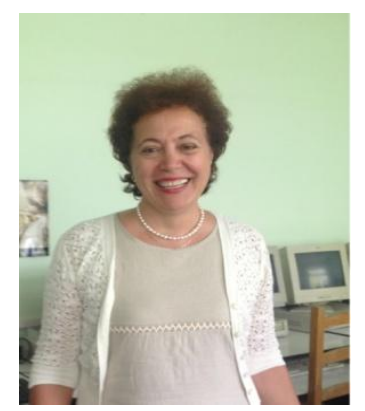

\section{Dr. Elida Hoxha (DOB-08/01/1961)}

Completed her M.Sc. in Mathematics from Tirana University in the year 1984 and completed her Ph.D. from Tirana University in 1997. She has a teaching experience of more than 29 years. Currently she is working as a Professor in the Department of Mathematics, Faculty of Natural Science, University of Tirana, Albania.

She is a popular teacher in under graduate and post graduate level. Her subject of teaching is Mathematical Analysis, Topology, Functional Analysis. Besides teaching she is actively engaged in research on the fields of Fixed Point Theory, Fuzzy sets and Fuzzy mappings, Topology. 